\title{
Physical Observable for One-dimensional Quantum Four-state Potts Model
}

\author{
Hong-Lei Wang ${ }^{1}$, Long-Cong Chen ${ }^{1}$, Bo Liang ${ }^{1}$, \\ ${ }^{1}$ Faculty of basic medical science, \\ Chongqing Medical University, \\ Chongqing 400016, P. R. China
}

\author{
Chun-Huan Xiang*2 \\ ${ }^{2}$ School of Public Health and Management, \\ Chongqing Medical University, \\ Chongqing 400016, P.R. China \\ *Corresponding author, E-mail: \\ xiang20122013@aliyun.com
}

\begin{abstract}
A one-dimensional quantum four-state Potts model is investigated in the context of a tensor network algorithm based on the infinite matrix product state representation. The local order parameter and entanglement entropy are computed. The scalling for the maximal entropy of the four-state Potts model is unveiled. The critical points are given, which is well agree with other know results.
\end{abstract}

Keywords-quantum phase transition; local order parameter; entanglement entropy; four-state Potts Model; central charge.

\section{INTRODUCTION}

In condense matter physics, quantum phase transitions imply fluctuations, which happened at the zero temperature [1]. In recent years, low-dimensional quantum magnets have received much attention in condensed matter physics. In particular, onedimensional(1D) quantum spin chains with competing interactions that show exotic physical properties have been extensively studied in the past decades. The Potts model plays an essential role in classical and quantum statistical mechanics. The q-state Potts model $[2,3]$ is a generalization of the Ising model that is the two state Potts model. Baxter [4] calculated the free energy of the Potts model at the critical temperature, and showed that the model has a continuous phase transition for $\mathrm{q} \leq 4$, and and has a first-order phase transition for $\mathrm{q}>4$ in 1973. The 1D quantum four-state Potts system, which is a critical quantum model, had been an important subject in many-body physics for a long time.

Numerical renormalization group methods obtained a first remarkable success with Wilson's solution of the Kondo problem [5], White's density matrix renormalization group algorithm [6], are solidly established as the dominant computational approach to quantum lattice systems in one spatial dimension.

Recently the advances in classical simulations of quantum lattice systems in one spatial dimension are given [7-11], and the tensor network representation of quantum many body wave functions provides an efficient way to classically simulate quantum many body systems. The algrithm exploit two facts: invariance under translations of the system and parallelizability of local updates in the time-evolving block decimation algorithm, which are not contaminated by finite size corrections or boundary effects.

In this paper, the local order parameter, the probability distribution for degenerate states of the four-state Potts model and the scalling of entanglement entropy in the four-state Potts model are investigated.

\section{THE TIME-EVOLVING BLOCK DECIMATION ALGORITHM}

The time-evolving block decimation algorithm is employed to simulate time evolution for a quantum system. The computational cost of a simulation using tensor network algorithms is roughly proportional to the size of the lattice. However, when the system is invariant under translations, this cost can be made independent of the system's size [7-11]. The initial input state $\left|\Psi_{0}\right\rangle$ in the matrix formation, each bond index of the wave function is related to the Schmidt decomposition:

$$
|\Psi\rangle=\sum_{\alpha=1}^{\chi} \lambda_{\alpha}\left|\Phi_{\alpha}^{L}\right\rangle \otimes\left|\Phi_{\alpha}^{R}\right\rangle
$$

where $\lambda$ is the diagonal matrix, which contains the decreasingly ordered Schmidt coefficients, $\alpha$ labels the Schmidt vectors, which form orthonormal sets $\left\langle\Phi_{\alpha}^{L} \mid \Phi_{\alpha^{\prime}}^{L}\right\rangle=\left\langle\Phi_{\alpha}^{R} \mid \Phi_{\alpha^{\prime}}^{R}\right\rangle=\delta_{\alpha \alpha^{\prime}}$. By using absorb the unitary gate and singular value decomposition, the wave function is updated, the approximation ground state is obtained with the imaginary time evolution at last[7-11]. 


$$
\left|\Psi_{\tau}\right\rangle=\frac{\exp (-H \tau)\left|\Psi_{0}\right\rangle}{\| \exp (-H \tau)\left|\Psi_{0}\right\rangle \|}
$$

\section{THE FOUR-STATE POTTS MODEL AND THE PROBABILITY DISTRIBUTION OF THE DEGENERATE STATES}

The Hamiltonian for the 1D quantum Potts model [12, $13]$ is described as:

$$
H=-\sum_{i=-\infty}^{\infty}\left(\sum_{a=1,2,3} Q_{x, a}^{[i]} Q_{x, 4-a}^{[i+1]}+h Q_{z}^{[i]}\right),
$$

where $h$ is the transverse magnetic field, the Potts matrix $Q_{a, x}(a=1,2,3)$ meet the relationship: $Q_{1, x} Q_{2, x}=Q_{3, x}, Q_{2, x} Q_{3, x}=Q_{1, x}, \quad Q_{3, x} Q_{1, x}=Q_{1, x} Q_{3, x}$. The Potts spin matrixs are given as

$$
\begin{gathered}
Q_{x, 1}=\left(\begin{array}{cccc}
0 & 1 & 0 & 0 \\
0 & 0 & 1 & 0 \\
0 & 0 & 0 & 1 \\
1 & 0 & 0 & 0
\end{array}\right) ; Q_{x, 2}=\left(\begin{array}{cccc}
0 & 0 & 1 & 0 \\
0 & 0 & 0 & 1 \\
1 & 0 & 0 & 0 \\
0 & 1 & 0 & 0
\end{array}\right) ; Q_{x, 3}=\left(\begin{array}{llll}
0 & 0 & 0 & 1 \\
1 & 0 & 0 & 0 \\
0 & 1 & 0 & 0 \\
0 & 0 & 1 & 0
\end{array}\right) ; \\
Q_{z}=\left(\begin{array}{cccc}
3 & 0 & 0 & 0 \\
0 & -1 & 0 & 0 \\
0 & 0 & -1 & 0 \\
0 & 0 & 0 & -1
\end{array}\right)
\end{gathered}
$$

The exact values for the scaling and critical exponents $[3,14]$ of the four-state Potts model are $\alpha=2 / 3 ; \beta=1 / 12$; $\gamma=7 / 6 ; \delta=15 ; v=2 / 3$. The critical point is $h=1$, and the central charge from comform field theory is $c=1$.

Like the 1D quantum Ising chains, the four-state quantum Potts model has two phases: a disordered phase for sufficiently large values of $h>1$ and an ordered phase for small $h<1$.

The 1D quantum four-state Potts model have four degenerated ground state, that is to say the $q=4$ for this model. By employing the time-evolving block decimation algorithm, which automatically produces degenerate ground states, the approximation ground states are obtained, where the initial state is given randomly. The total number of the approximation ground states is 6000 with the transverse magnetic field $h=0.4$, which is given in the spontaneous symmetry breaking section, where the four-degenerated state is appeared in equal probability. The truncation dimension is $\chi=4$. We labeled one of the degenerated state when the data of $N$ degenerated states are presented, $N=80,120,160$, respectively. The number for picking up some of the ground state is 100000 times. This confirms that the probability of getting the ground state is $1 / 4$. The probability mass function is obtained finally, which is shown in Fig. 1. We expect that this is true for any model with degenerated four-state.

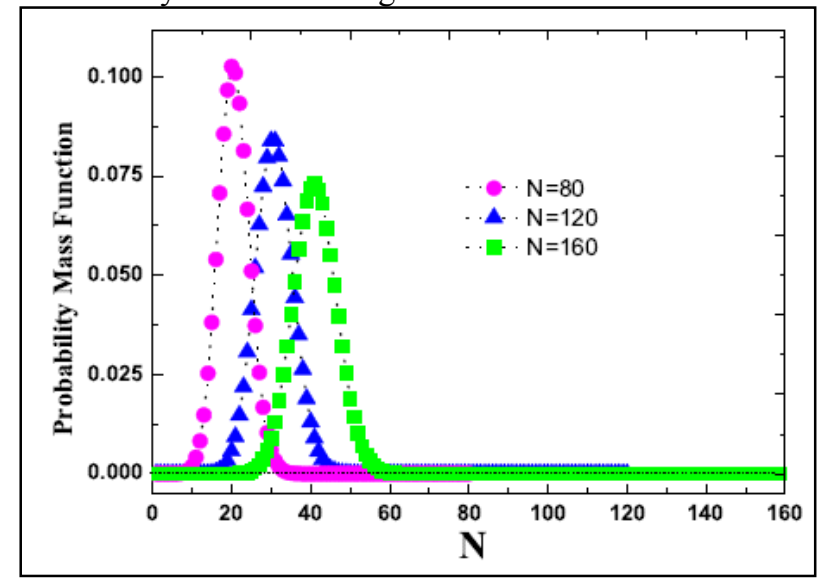

Fig.1. The probability mass function for one-dimensional quantum fourstate Potts model. The total number of the ground state for the model is 6000 with the transverse magnetic field $h=0.4$, the data are presented $N$ ground states, and one of the degenerated state is label. The number for pick up some of the ground state is 100000 . The maximal probability for $N=80,120,160$ is $20,30,40$, respectively. The probability for any of the degenerated four- state is equal, which is $1 / 4$.

\section{SIMULATION RESULTS}

The local order parameter is one of the most important physical observable. The time-evolving block decimation algorithm makes it possible to extract a local order parameter. The single-site reduced density matrix is obtained from the approximation ground-state. When the transverse magnetic field $h$ runs from the $h>h_{\mathrm{c}}$ to $h<h_{\mathrm{c}}, h_{\mathrm{c}}$ $=1$, the parameter $<\mathrm{M}>=<\mathrm{Q}_{\mathrm{x}, 1}+\mathrm{Q}_{\mathrm{x}, 2}+\mathrm{Q}_{\mathrm{x}, 3}>/ 3$ as the local order parameter is obtained. The local order parameter $<\mathrm{M}>$ is nonzero with the transverse magnetic field $h$ in the spontaneous symmetry breaking section, which is zero in the symmetry phase. The figure is shown in Fig. 2, with matrix dimension $\chi=8,16,32,64$, respectively. The critical point given by $\chi=64$ is $h=1.001$. 


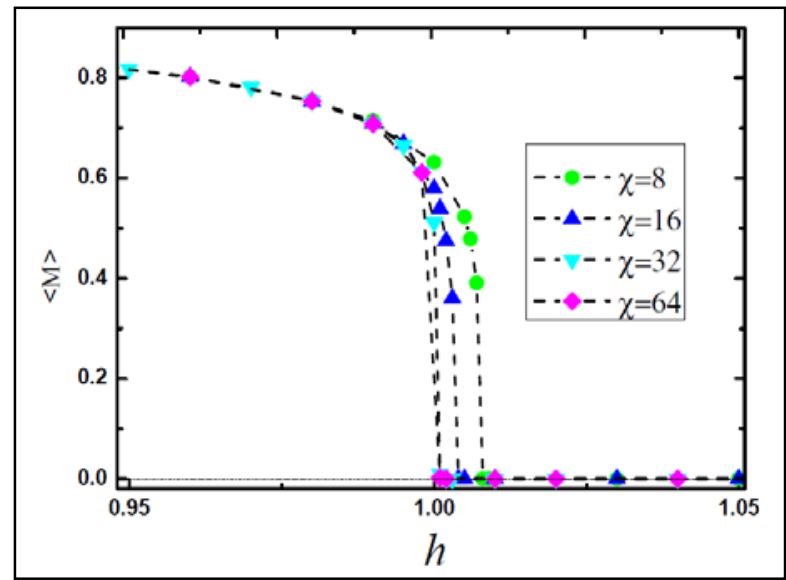

Fig.2. The local order parameter for one-dimensional quantum fourstate Potts model. The local order paramete $<\mathrm{M}>$, which is the function of the transverse magnetic field $h$, is shown with matrix dimension $\chi=8$,

$16,32,64$, respectively. The critical point given by the trunction dimension is getting closer and closer to the exact critical point $h=1$. The spontaneous symmetry breaking is occurred with the transverse magnetic field $h<1$.

The von Neumann entropy is a measure of a bipartite entanglement present in a quantum state, which is also used to detect the quantum phase transition point. We may try to find the exact amount of entanglement which is captured. The entanglement entropy can be measured by the entanglement entropy as

$$
\mathrm{S}=-\operatorname{Tr} \lambda^{2} \log \lambda^{2}
$$

where $\lambda$ is a diagonal matrix. Time-evolving block decimation algorithm is automatically produce the diagonal matrix $\lambda$ and tensor $\Gamma$ in the matrix product states at last. The peak of the entanglement entropy increases with the matrix dimension increases. It diverges logarithmically at a quantum transition point with matrix dimension $\chi \rightarrow \infty$. We have found that the quantitative entanglement entropy support of time-evolving block decimation algorithm at criticality obeys the following scaling law [14-17]

$$
\mathrm{S}=\mathrm{a} \log (\chi)+\mathrm{b}
$$

where $a$ and $b$ are parameters to be determined, $S$ is the maximal entanglement entropy for the trunction dimension. The relationship is shown in Fig. 3, the matrix dimension $\chi=4,6,8,12,16,32$, respectively. The parameters are obtained with $a=0.1606, b=0.4630$.

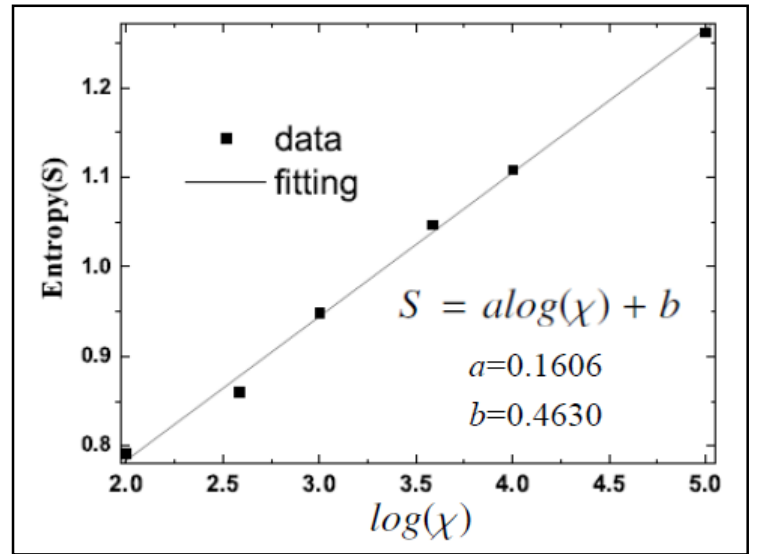

Fig.3. The scalling relationship of the entanglement entropy $S$ with $\log (\chi)$ for the one-dimensional quantum four-state Potts model. The scalling low is $S=a \log (\chi)+b$, the parameters $a$ and $b$ to be determined. The maximal entropies for matrix dimensions $\chi=4,6,8,12,16,32$ are shown. The parameters are obtained with $a=0.1606$ and $b=0.4630$.

\section{CONCLUSIONS}

All our numerical results were found using the timeevolving block decimation algorithm. The probability mass function, local order parameter and the scalling of entanglement entropy with the matrix dimension are obtained. We confirmed the degenerated four-state of the 1D quantum Potts model in a transverse magnetic field with numerical simulation. The critical point obtained by local order parameter $<\mathrm{M}>=<\mathrm{Q}_{\mathrm{x}, 1}+\mathrm{Q}_{\mathrm{x}, 2}+\mathrm{Q}_{\mathrm{x}, 3}>/ 3$ is agree with the exact critical point $h=1$. The scaling of entanglement entropy with the matrix dimension $\chi$ can be used to analyze properties of critical models with relatively small effort. We expect finite- $\chi$ scaling to appear for some generalizations of matrix product states. The quantum phase transitions for $q$-state Potts model is an interesting issue which calls for future investigations.

\section{ACKNOWLEDGEMENTS}

This work was financially supported by the National Natural Science Foundation of China (Grant No: 11447115) and the Science Foundation of Chongqing City Board of Education (KJ121206). 


\section{REFERENCES}

[1] Sachdev S. Quantum Phase Transitions. Cambridge: Cambridge University Press, 1999.

[2] Potts R. B. Q-state Potts models in Hamiltonian field theory for Q $\geq 4$ in $(1+1)$ dimensions, Process Cambridge philosophy Society, 48 , 106, 1952.

[3] Wu F. Y., The Potts Model, Review Modern Physics, 54, 235, 1982.

[4] Baxter R. J., Critical Exponents of the Four-State Potts Model, Journal of Physics: Condensed Matter, 6, L445, 1973.

[5] K. Wilson, Renormalization Group and the Kadanoff Scaling Picture, Physical Review B, 4, 3184, 1971.

[6] White S. R., Density matrix renormalization group, Physical Review Letters, 69, 2863, 1992.

[7] Vidal G., Classical simulation of infinite-size quantum lattice systems in one spatial dimension, Physical Review Letters, 98, $070201,2007$.

[8] Vidal G., Efficient classical simulation of slightly entangled quantum computations, Physical Review Letters, 91, 147902, 2003.

[9] Vidal G., Efficient simulation of one-dimensional quantum manybody systems, Physical Review Letters, 93, 040502, 2004.

[10] White S. R., Feiguin A. E., Real time evolution using the density matrix renormalization group, Physical Review Letters, 93, 076401 2004.

[11] Daley A. J., Kollath C., Schollwoeck U., Vidal G., Time-dependent density-matrix renormalization-group using adaptive effective Hilbert spaces, Journal of Statistical Mechanics: Theory and Experiment, P04005, 2004.

[12] Enting I. Critical exponents for the four-state Potts model, Journal of Physics A: Mathematical and General, 8, L35-L38, 1975.

[13] Foster D. and G'erard C. The ferro/antiferromagnetic $q$-state Potts model, Journal of Physics A: Mathematical and General, 35, L75L80, 2002.

[14] Taddia1 L., Xavier J. C., Alcaraz F. C. and Sierra G. Entanglement Entropies in Conformal Systems with Boundaries, Physical Review B, 88, 075112, 2013.

[15] Lepori L., Chiara G., and Sanpera A., Scaling of the entanglement spectrum near quantum phase transitions, Physical Review B, 87, 235107, 2013.

[16] Chu R.-L., Zhao A., and Shen S.-Q., Finite size scaling of entanglement entropy at the Anderson transition with interactions, arxiv: 1210.2470 .

[17] Rodney M., Song H., Lee S.-S., Hur K. and Srensen E., Scaling of entanglement entropy across Lifshitz transitions, Physical Review B, 87, 1151322013. 